\title{
Prevention of exacerbations in patients with stable non-cystic fibrosis bronchiectasis: a systematic review and meta-analysis of pharmacological and non-pharmacological therapies
}

\author{
Abd Moain Abu Dabrh, ${ }^{1,2}$ Adam T Hill, ${ }^{3}$ Claudia C Dobler, ${ }^{1}$ \\ Noor Asi, ${ }^{1}$ Wigdan H Farah, ${ }^{1}$ Qusay Haydour, ${ }^{1}$ Zhen Wang, ${ }^{1}$ \\ Khalid Benkhadra, ${ }^{1,4}$ Larry J Prokop, ${ }^{5}$ \\ Mohammad Hassan Murad ${ }^{1}$
}

\subsection{6/bmjebm-2018-110893}

- Additional material is published online only. To view please visit the journal online (http://dx.doi.org/ 10.1136/bmjebm-2018110893).

${ }^{1}$ Evidence-based Practice Center, Robert D and Patricia E Kern Center for the Science of Health Care Delivery, Mayo Clinic, Rochester, Minnesota, USA

${ }^{2}$ Department of Family Medicine, Mayo Clinic, Jacksonville, FL, USA

${ }^{3}$ Department of Respiratory Medicine, Royal Infirmary and University of Edinburgh, Edinburgh, UK ${ }^{4}$ Internal Medicine, School Of Medicine Wayne State University, Detroit, Michigan, USA

${ }^{5}$ Library Public Services, Mayo Clinic, Rochester, Minnesota, USA

Correspondence to: Abd Moain Abu Dabrh, Department of Family Medicine, Mayo Clinic, Jacksonville FL 32224, USA; abudabrh.abdmoain@mayo. edu

Check for updates

To cite: Abu Dabrh AM, Hill AT, Dobler CC, et al. BMJ Evidence-Based Medicine 2018;23:96-103.

\section{Abstract}

Background Several pharmacological and nonpharmacological therapies are used to treat stable bronchiectasis of non-cystic fibrosis (CF) aetiology. Objective We conducted a systematic review and meta-analysis to assess the evidence of the effectiveness of pharmacological and nonpharmacological treatment options in patients with stable non-CF bronchiectasis with a focus on reducing exacerbations.

Study selection Multiple databases were searched through September 2017. Outcomes included the number of patients with exacerbation events, mean number of exacerbations, hospitalisations, mortality, quality of life measures, and safety and adverse effects. Meta-analysis was conducted using the random effects model.

Findings 30 randomised controlled trials enrolled subjects with non-CF bronchiectasis using different interventions. Moderate-quality evidence supported the effect of long-term antibiotics ( $\geq 3$ months) on lowering the number of patients experiencing exacerbation events (relative risk 0.77 (95\% CI 0.68 to 0.89)), reducing number of exacerbations (incidence rate ratio 0.62 (95\% CI 0.49 to 0.78)), improving forced expiratory volume (litre) in the first second $\left(\mathrm{FEV}_{1}\right)$ (weighted mean difference (WMD); 0.02 (95\% CI 0.00 to 0.04)), decreasing sputum purulence scores (numerical scale of 1-8) (WMD -0.90 (95\% CI -1.58 to -0.22$)$ ) and improving quality of life scores assessed by the St George's Respiratory Questionnaire (WMD -6.07 (95\% CI - 10.7 to -1.43)). Bronchospasm increased with inhaled antibiotics while diarrhoea increased particularly with oral macrolide therapy.

Conclusions Moderate-quality evidence supports long-term antibiotic therapy for preventing exacerbations in stable non-CF bronchiectasis. However, data about the optimum agent, mode of therapy and length of treatment are limited. There is paucity of high-quality evidence to support the management of stable non-CF bronchiectasis including prevention of exacerbations.

\section{Introduction}

Bronchiectasis is a chronic respiratory condition, often characterised by daily cough and chronic sputum production with a predisposition to recurrent chest infections. The global prevalence of bronchiectasis is unknown but it is being diagnosed more commonly in recent times, perhaps because high-resolution chest CT scanning-the gold standard for diagnosis-is more frequently used. In the USA, the prevalence ranges from 4 to 300/100 000 in adults. ${ }^{12}$ Severity may be assessed using different scoring systems, including the validated FACED and Bronchiectasis Severity Index (BSI) scores. ${ }^{34}$ The BSI has been shown to be associated with risk of hospitalisation and mortality.

Up to 55\% of cases are idiopathic (ranges from $25 \%$ to $53 \%$ from studies with $>100$ patients with bronchiectasis) and the second most common cause is a past respiratory infection $(4 \%-42 \%){ }^{1}$ Cole coined the phrase 'the vicious cycle' when describing the pathogenesis of bronchiectasis. ${ }^{5} 6$ Pharmacological therapies designed to break this cycle can be grouped into anti-infective, anti-inflammatory and mucoactive agents. Treatment aims to reduce the number of exacerbations, reduce cough and sputum volume, reduce sputum purulence and improve health-related quality of life.

This systematic review and meta-analysis assesses the evidence of different therapies by providing a comprehensive overview of the effectiveness of pharmacological and non-pharmacological treatment options in patients with stable bronchiectasis of non-cystic fibrosis (CF) aetiology.

\section{Methods}

This review followed a priori protocol with consensus and clinical expertise input from members of the American College of Chest Physicians (CHEST) Expert Cough Panel regarding the interventions (online supplementary table 1) and outcomes of interest. The reporting of this review follows the Preferred Reporting Items for Systematic Reviews and Meta-Analyses statement. ${ }^{7}$

\section{Study eligibility and literature search}

Studies included randomised controlled trials (RCT), including crossover trials that enrolled patients with stable bronchiectasis of non-CF aetiology and compared interventions of interest 
to placebo, no intervention, other different interventions or to inhaled saline. We extracted data on patient demographics, baseline characteristics, study design variables, sample size, length of follow-up, intervention types, number of patients in each intervention and outcomes of interest. The search strategy is further detailed in the online supplementary material.

\section{Outcomes}

For exacerbations, we reported the number of patients who were at risk and experienced protocol-defined exacerbations (PDE). We also reported the mean number of PDEs. Additionally, we have reported the number of hospitalisations, all-cause mortality and scale-based sputum purulence. We evaluated the quality of life as assessed by St George's Respiratory Questionnaire (SGRQ). ${ }^{8}$ We also reported the impact of cough as assessed by the validated Leicester Cough Questionnaire (LCQ). ${ }^{10}$ We also extracted different clinically important and commonly reported intervention-pertinent adverse events (AE). Definitions of all these outcomes are detailed in the online supplementary material.

\section{Risk of bias and quality of evidence assessment}

We used the Cochrane Collaboration Risk of Bias Assessment Tool. ${ }^{11}$ We graded the strength of evidence using the Grades of Recommendation, Assessment, Development and Evaluation workgroup methodology. ${ }^{12}$

\section{Statistical analysis}

For patients with exacerbations and exacerbation events, we extracted and reported the discrete number of events and total sample. Reported scales were extracted as pre-post mean differences. For dichotomised outcomes, we calculated or extracted relative risks (RR). For exacerbation events (incidence) and some predefined safety and diagnostic endpoint outcomes, we used incidence rate ratio (IRR). The DerSimonian and Laird random effects methods were used to pool outcomes from the included studies. For continuous outcomes, we pooled weighted mean difference (WMD) using the same random effects model. For crossover RCTs, we extracted means and SDs (or SEs) for the intervention and the control for the whole study period. This approach is likely to overestimate the 'true' SEs and have wide CIs for crossover RCTs; thus, those studies contributed 'less' in the pooled effect size (ie, less weights) using the D-L random effects model. We conducted the analysis accounting for the intention-to-treat (ITT) population of the included studies. If the analysis in the original study was not based on ITT, we recalculated outcomes using ITT analysis where the study information provided was sufficient. We conducted subgroup analyses to explore heterogeneity between studies based on predefined effect modifiers. We statistically evaluated the difference between subgroups using one-way analysis of variance test. Heterogeneity across the included studies was estimated using $\mathrm{I}^{2}$ statistic, in which $\geq 50 \%$ suggests substantial heterogeneity. We were unable to assess publication bias due to the small number of studies included in each analysis and substantial heterogeneity. Statistical analyses were completed using Stata V.12 (StataCorp, College Park, TX).

\section{Results}

The initial search yielded 1050 publications, and eventually 30 studies were included as the flow chart shows in online supplementary eFigure 1 . The characteristics of included studies are summarised in online supplementary table 2 while online supplementary table 3 reflects the risk of bias assessment. The risk of bias across outcomes and in most of the interventions was moderate mainly due to unclear allocation concealment. Results of the meta-analysis of primary and safety outcomes are shown in tables 1-3 and online supplementary tables 4 and 5. Only three studies reported the endpoints of interest in children. ${ }^{13-15}$ The most common interventions reported were antibiotics versus placebo (17 studies), ${ }^{13-29}$ mucoactive agents (rhDNase+mannitol) versus placebo (three studies ${ }^{30-32}$; two studies about mannitol only ${ }^{30} 31$ vs placebo+onestudy ${ }^{32}$ about rhDNase vs placebo), inhaled corticosteroids versus placebo (three studies), ${ }^{3-35}$ pulmonary rehabilitation (PR; three studies), ${ }^{36-38}$ physiotherapy (PT) versus no $\mathrm{PT}_{ \pm}$usual care (two studies) ${ }^{39} 40$ and statins (one study). ${ }^{41}$ There was paucity in studies reporting outcomes of interest comparing for several interventions of interest, including patients who received surgery, certain vaccines or gastro-oesophageal reflux disease (GORD/GERD) treatments. Certain interventions or outcomes were only reported using single trial results (table 3 and online supplementary table 5).

Twenty-one studies of different interventions used various outcome-specific indices or quality of life scales, with the most common reported scales being SGRQ ${ }^{14} 1621232627293133-384042$ and $\mathrm{LCQ}^{23} 26363740$ (both quality of life scales), and Sputum Purulence Scale (measurement of sputum colour). ${ }^{14}{ }^{34}$ A summary of some of the reported or used scales pertinent to the outcomes of interested is shown in online supplementary table 6 . The main primary outcomes of the included studies are summarised in online supplementary table 7. We included four crossover RCTs in the analyses. All of them reported adequate washout period to exclude possible effects from the precrossover phase.

\section{Results of meta-analysis}

\section{Antibiotics versus placebo}

Seventeen studies ${ }^{13-29}$ reported different outcomes comparing antibiotics versus placebo (tables 1 and 2). Thirteen studies enrolling 1466 patients reported number of patients with exacerbations. ${ }^{13-18} 2022$ 23 25-28 The use of antibiotic was associated with a lower proportion of patients experiencing exacerbations when compared with those who received placebo (RR $0.76(0.65,0.89)$ ) (table 1, figure 1).

Neither the age group of patients nor the route of antibiotic administration showed statistically significant difference (table 1).

Fourteen studies enrolling 1196 patients reported frequency of exacerbations (mean number of events). $.^{13} 15-21232426-29$ When compared with placebo, the use of antibiotics was significantly associated with decrease in the number of exacerbations in patients with bronchiectasis (IRR $0.62(0.49,0.78)$ ) (table 1, figure 2). There was no significant difference between other subgroups.

Five studies enrolling 805 patients reported patient hospitalisations. ${ }^{1617212427}$ There was no significant difference between the treatment groups (table 1, online supplementary eFigure 2). Six studies ${ }^{17} 21-232628$ enrolling 916 patients reported about mortality and found no significant difference with the use of antibiotics when compared with placebo (table 1, online supplementary eFigure 3).

In assessing measurement scales, studies using a Sputum Purulence Scale ${ }^{14} 34$ showed a statistically significant difference (ie, decrease in purulence, or improvement) as did studies measuring improvement in quality of life (total SGRQ) ${ }^{16} 20-22262729$ while using antibiotic versus placebo, (WMD $-0.90(-1.58,-0.22)$ ) and (WMD $-6.07(-10.7,-1.43)$ ) respectively (online supplementary table 5). All safety and adverse effect outcomes are summarised in table 2 .

In general, we found insufficient or unclear reporting of safety outcomes. Patients who received inhaled antibiotics had more than double the risk of bronchospasm (online supplementary 


\begin{tabular}{|c|c|c|c|c|c|c|}
\hline Outcome & Number of studies & Patients (n) & Effect size & $95 \% \mathrm{Cl}$ & $1^{2}(\%)$ & $\begin{array}{l}\text { P value } \\
\text { (interaction) }\end{array}$ \\
\hline \multicolumn{7}{|l|}{ Patients with exacerbations* } \\
\hline Total & 13 & 1466 & 0.76 & 0.65 to 0.89 & 58.4 & \\
\hline Adult studies & $9 \dagger$ & 1187 & 0.75 & 0.62 to 0.92 & 67.4 & 0.26 \\
\hline Paediatric studies & $3+$ & 157 & 0.86 & 0.73 to 1.01 & 0 & \\
\hline Route of administration (inhaled) & 4 & 791 & 0.82 & 0.60 to 1.10 & 64.8 & 0.53 \\
\hline Route of administration (oral) & 9 & 604 & 0.72 & 0.58 to 0.88 & 68.6 & \\
\hline Type of antibiotic (oral macrolides) & 7 & 444 & 0.69 & 0.53 to 0.91 & 69.5 & 0.84 \\
\hline Type of antibiotic (oral non-macrolides) & 2 & 160 & 0.76 & 0.41 to 1.40 & 80.5 & \\
\hline \multicolumn{7}{|l|}{ Exacerbations (mean) $\ddagger$} \\
\hline Total & 14 & 1196 & 0.62 & 0.49 to 0.78 & 81.1 & \\
\hline Adult studies & $11 \dagger$ & 603 & 0.55 & 0.40 to 0.75 & 83.4 & 0.63 \\
\hline Paediatric studies & $2 \dagger$ & 132 & 0.67 & 0.37 to 1.22 & 78 & \\
\hline Route of administration (inhaled) & 5 & 826 & 0.67 & 0.44 to 1.02 & 81.9 & 0.68 \\
\hline Route of administration (oral) & 8 & 653 & 0.47 & 0.38 to 0.59 & 31.9 & \\
\hline Type of antibiotic (oral macrolides) & 7 & 520 & 0.49 & 0.39 to 0.55 & 28.4 & 0.76 \\
\hline Type of antibiotic (oral non-macrolides)§ & 1 & 38 & 0.29 & 0.14 to $0.55 \S$ & - & \\
\hline Pseudomonas aeruginosa & 3 & 189 & 0.70 & 0.44 to 1.12 & 0 & - \\
\hline \multicolumn{7}{|l|}{ Mortality* } \\
\hline Total & 6 & 916 & 1.25 & 0.49 to 3.18 & 0 & - \\
\hline \multicolumn{7}{|l|}{ Number of hospitalisation $\ddagger$} \\
\hline Total & 5 & 805 & 0.51 & 0.22 to 1.13 & 45.2 & - \\
\hline
\end{tabular}

*Relative risk.

tDrake and Knowelden ${ }^{28}$ enrolled undifferentiated mixed population; therefore, it was excluded from this subgroup analysis.

‡Incidence rate ratio.

§Unadjusted; Currie et $\mathrm{al}^{18}$ reported no statistical significance after adjustment to frequency of exacerbations reported in the prior year to enrolment.

eFigure 4) while the risk of diarrhoea tripled with oral antibiotics when compared with placebo (table 2). Also, patients who received antibiotics, ${ }^{15} 1827$ especially inhaled antibiotics, ${ }^{15} 27$ had an almost 2.5-fold increased risk of withdrawal from treatment due to toxicity or adverse effects (RR 2.47 (1.29, 4.71)) (table 2).

Patients who received antibiotics, specifically oral macrolides, showed significant improvement in forced expiratory volume in $1 \mathrm{~s}\left(\mathrm{FEV}_{1}\right)(\mathrm{WMD} 0.02(0.00,0.04))$ (online supplementary eFigure 5). Different routes of antibiotic use (oral macrolides vs inhaled antibiotics) did not show any statistically significant differences in effectiveness. ${ }^{18-20} 22$ 24-27 Patients who received inhaled antibiotics had a statistically significant reduction in their forced vital capacity (FVC)\% predicted when compared with those who were on oral antibiotics (WMD $-3.85(-5.02,-2.67)$ vs $0.23(-1.78$, $-2.24)$ ). The quality of evidence comparing antibiotics versus placebo is likely low to moderate, graded down due to heterogeneity and increased risk of bias.

\section{Inhaled corticosteroids versus placebo}

Two studies ${ }^{33}{ }^{34}$ enrolling 163 patients investigated the number of patients with exacerbation and found no significant difference (OR $0.85(0.63,1.14)$ ) (table 3 , figure 1). Similarly, these studies $^{33}{ }^{34}$ reported exacerbation events (mean), and found that when compared with placebo, inhaled corticosteroids did not significantly reduce exacerbations (table 3, figure 2).

Number of hospitalisations and mortality were both not significantly different between inhaled corticosteroids and placebo. The measurement scales (online supplementary table 5) showed only statistically significant deterioration on the total SGRQ measure of quality of life.
Lung function showed a small improvement in $\mathrm{FEV}_{1} \%$ predicted $^{33} 34$ and deterioration in FVC\% predicted ${ }^{33-35}$ but no significant effect on the absolute value of $\mathrm{FEV}_{1}^{35}$ with the use of inhaled corticosteroids. There were no reported occurrences of pneumonia in both groups in the two included studies. Generally, there was sparse or insufficient reporting of safety outcomes; one study reported no significant differences in haemoptysis and sore throat. There was paucity in reporting other significant outcomes, including adrenal suppression, skin thinning and bone mineral density. All safety and adverse effect outcomes are summarised in online supplementary table 4. The quality of evidence comparing inhaled corticosteroids versus placebo is low, graded down due to imprecision and increased risk of bias.

\section{Inhaled corticosteroids versus inhaled corticosteroids+long-acting $\beta 2$ agonist}

No statistically significant differences were observed with exacerbations or the quality of life measures as reported in 40 patients ${ }^{42}$ (table 3 ). The quality of evidence is low, graded down due to severe imprecision.

\section{Mucoactive versus placebo}

Three studies enrolled 1177 patients who received any type of mucoactive agent. ${ }^{30-32}$ One study ${ }^{32}$ enrolled 349 patients receiving rhDNase while two studies ${ }^{30} 31$ enrolled 828 patients receiving mannitol only in comparison to control or placebo treatments. Mucoactive agents (rhDNase) only showed a statistically significant decline in lung function measured by $\mathrm{FEV}_{1}$ (\% predicted) and FVC (\% predicted). O'Donnell et $a l^{32}$ reported three different counts of exacerbation events using PDE, non-PDE and combined 


\begin{tabular}{|c|c|c|c|c|c|c|}
\hline Outcome & Number of studies & Patients (n) & Effect size & $95 \% \mathrm{Cl}$ & $1^{2}(\%)$ & $P$ value (interaction) \\
\hline \multicolumn{7}{|l|}{ Bacterial resistance* } \\
\hline Total & 7 & 444 & 1.68 & 0.70 to 4.07 & 60.6 & \\
\hline Route of administration (inhaled) & 3 & 87 & 1.03 & 0.23 to 4.70 & 43.1 & 0.11 \\
\hline Route of administration (oral) & 4 & 357 & 2.31 & 0.65 to 8.19 & 75 & \\
\hline Type of antibiotic (oral macrolides) & 3 & 319 & 2.23 & 0.049 to 10.15 & 82.2 & 0.71 \\
\hline Type of antibiotic (oral non-macrolides) & 1 & 38 & 3 & 0.34 to 26.33 & - & \\
\hline \multicolumn{7}{|l|}{ Bronchospasm* } \\
\hline Total & 7 & 861 & 2.07 & 1.06 to 4.01 & 0 & \\
\hline Adult patients+inhaled antibiotics studies & 7 & 861 & 2.07 & 1.06 to 4.01 & 0 & \\
\hline \multicolumn{7}{|l|}{$\mathrm{FEV}_{1}(\mathrm{~L}) \dagger$} \\
\hline Total & 7 & 500 & 0.02 & 0.00 to 0.04 & 0 & \\
\hline Route of administration (inhaled) & 3 & 201 & 0.04 & -0.02 to 0.09 & 0 & 0.47 \\
\hline Route of administration (oral macrolides) & 4 & 299 & 0.02 & 0.00 to 0.04 & 0 & \\
\hline \multicolumn{7}{|l|}{$\mathrm{FEV}_{1}$ (predicted, \%)† } \\
\hline Total & 9 & 674 & -0.10 & -0.22 to 0.01 & 0 & \\
\hline Route of administration (inhaled) & 4 & 259 & 0.16 & -1.65 to 1.96 & 55.1 & 0.26 \\
\hline Route of administration (oral macrolides) & 5 & 415 & 0.15 & -1.68 to 1.96 & 0 & \\
\hline \multicolumn{7}{|l|}{ FVC (predicted, \%)† } \\
\hline Total & 6 & 344 & -1.21 & -4.19 to 1.78 & 98.1 & \\
\hline Route of administration (inhaled) & 3 & 130 & -3.85 & -5.02 to 2.67 & 10.7 & 0.000002 \\
\hline Route of administration (oral macrolides) & 3 & 214 & 0.23 & -1.78 to 2.24 & 8.2 & \\
\hline \multicolumn{7}{|l|}{ Diarrhoea* } \\
\hline Total & 5 & 401 & 3.04 & 1.63 to 5.68 & 0 & \\
\hline Type of antibiotic (oral macrolides) & 3 & 241 & 4.23 & 1.72 to 10.38 & 0 & 0.36 \\
\hline Type of antibiotic (oral non-macrolides) & 2 & 160 & 1.67 & 0.45 to 6.19 & 0 & \\
\hline \multicolumn{7}{|l|}{ Nausea* } \\
\hline Total & 4 & 385 & 1.07 & 0.58 to 1.97 & 0 & \\
\hline \multicolumn{7}{|l|}{ Withdrawal due to toxicity or $\mathrm{AE}^{*}$} \\
\hline Total & 12 & 1324 & 2.47 & 1.29 to 4.71 & 0 & \\
\hline Route of administration (inhaled) & 7 & 861 & 2.89 & 1.23 to 6.76 & 0 & 0.21 \\
\hline Route of administration (oral) & 5 & 463 & 1.06 & 0.29 to 3.81 & 0 & \\
\hline Type of antibiotic (oral macrolides) & 3 & 347 & 0.80 & 0.17 to 3.72 & 0 & 0.79 \\
\hline Type of antibiotic (oral non-macrolides) & 2 & 86 & 2 & 0.20 to 20.24 & - & \\
\hline
\end{tabular}

*Relative risk.

tWeighted mean difference.

$\mathrm{AE}$, adverse event; $\mathrm{FEV}_{1}$, forced expiratory volume in $1 \mathrm{~s}$; FVC, forced vital capacity.

(PDE+non-PDE) events; using the PDE showed no significant association while using data from the combined event yielded a weak statistical significance with increased incidence of exacerbations (1.35; 95\% CI 1.01 to 1.79 ).

All studies whether comparing the combined mucoactive agent studies, mannitol only (two studies) or rhDNase only (one study), were not associated with any significant difference in other outcomes when compared with placebo (table 3, figures 1 and 2 , and online supplementary eFigures 3 and 4). The quality of evidence is low, graded down due to imprecision and increased risk of bias. All safety and adverse effect outcomes are summarised in online supplementary table 4 .

\section{PT and PR}

We identified two studies of PT: using oscillatory positive expiratory pressure (PEP) device in one study ${ }^{40}$ and using flutter device in another study. ${ }^{39}$ Receiving PT alone was associated with better quality of life (SGRQ total component and LCQ scales) when compared with no PT care ${ }^{40}$ (online supplementary table 5). There were not sufficient data available about other outcomes (table 3). The quality of evidence is low, graded down due to imprecision and increased risk of bias.
PR is a programme of education, exercise and support for patients to breathe better and function at the highest possible capacity in their daily lives. We identified three studies. ${ }^{36-38}$ One study ${ }^{36}$ used a specialised chest physiotherapist to train the enrolled patients. Once the physiotherapist was satisfied with the patient technique, chest PT was done at home but was checked at each study visit. Another study compared PR with a targeted inspiratory muscle training (IMT) versus PR+Sham IMT versus control care. ${ }^{38}$ The third study compared a supervised exercise training and educator-supported group versus physical education-only group. ${ }^{37} \mathrm{PR}$, coupled with PT, was associated with better quality of life (measured by SGRQ total) when compared with PT-only treatment. ${ }^{3637}$ The quality of evidence is low, graded down due to imprecision and increased risk of bias.

\section{Other interventions}

Atorvastatin was associated with a lower number of patients with exacerbations and exacerbation events (mean) while improving the quality of life (improvement in LCQ scores) when compared with placebo in one study ${ }^{41}$ (figure 1, table 3 and online supplementary table 5). 
Table 3 Outcomes of various predefined interventions in stable patients with bronchiectasis

\begin{tabular}{|c|c|c|c|c|c|c|}
\hline Outcome & Interventions & Number of studies & Patients (n) & Effect size & $95 \% \mathrm{Cl}$ & $\mathrm{I}^{2}(\%)$ \\
\hline \multirow{4}{*}{$\begin{array}{l}\text { Patients with } \\
\text { exacerbations* }\end{array}$} & Corticosteroids (inhaled) versus placebo & 2 & 163 & 0.85 & 0.63 to 1.14 & 0 \\
\hline & $\begin{array}{l}\text { Corticosteroids (inhaled) versus corticosteroids } \\
\text { (inhaled)+long-acting } ß 2 \text { agonist }\end{array}$ & 1 & 40 & 1.75 & 0.61 to 5.05 & - \\
\hline & Mucoactive (mannitol) versus placebo & 2 & 828 & 0.89 & 0.79 to 1.00 & - \\
\hline & Atorvastatin versus placebo & 1 & 60 & 0.50 & 0.25 to 0.99 & - \\
\hline \multirow[t]{6}{*}{ Exacerbations (mean) $\dagger$} & Corticosteroids (inhaled) versus placebo & 2 & 163 & 0.81 & 0.63 to 1.04 & 0 \\
\hline & Mucoactive agents versus placebo & 3 & 1173 & 0.97 & 0.70 to 1.33 & 76.5 \\
\hline & rhDNase versus placebo & 1 & 349 & 1.17 & 0.88 to 1.53 & - \\
\hline & Mannitol versus placebo & 2 & 828 & 0.92 & 0.81 to 1.06 & 0 \\
\hline & $\begin{array}{l}\text { Corticosteroids (inhaled) versus corticosteroids } \\
\text { (inhaled)+long-acting } ß 2 \text { agonist }\end{array}$ & 1 & 40 & 1.75 & 0.43 to 7.02 & - \\
\hline & PT versus no PT (usual care) & 2 & 29 & 0.71 & 0.19 to 2.55 & 0 \\
\hline \multirow[t]{3}{*}{ Mortality* } & Corticosteroids (inhaled) versus placebo & 3 & 203 & 0.39 & 0.07 to 2.09 & - \\
\hline & Mucoactive versus placebo & 1 & 349 & 3.05 & 0.32 to 29.06 & 0 \\
\hline & Mannitol versus placebo & 2 & 828 & 0.69 & 0.06 to 8.18 & - \\
\hline \multirow{2}{*}{$\begin{array}{l}\text { Number of } \\
\text { hospitalisationt }\end{array}$} & Corticosteroids (inhaled) versus placebo & 2 & 163 & 0.61 & 0.16 to 2.31 & 0 \\
\hline & Mannitol versus placebo & 1 & 461 & 0.60 & 0.35 to 1.01 & - \\
\hline
\end{tabular}

*Relative risk.

tIncidence rate ratio.

PT, physiotherapy.

\section{Discussion}

This systematic review and meta-analysis identified criteria-specific therapies that could have a positive impact on exacerbations in patients with clinically stable bronchiectasis. The term "clinically stable' in its own right is debatable as although patients with bronchiectasis do not have a current exacerbation, many patients remain symptomatic with regular cough and sputum production, may have systemic symptoms and are prone to recurrent exacerbations. The number of patients with exacerbations was reduced by high-dose statin therapy and also with long-term antibiotic

\begin{tabular}{|c|c|c|c|}
\hline Study ID & Intervention Group & Comparison Group & \\
\hline \multicolumn{4}{|l|}{ Antibiotics } \\
\hline Masekela 2013 & Antibiotics (Erythromycin) & Placebo & \\
\hline Murray 2011 & Antibiotics (Gentamicin) & Placebo & $\rightarrow$ \\
\hline Altenburg 2013 & Antibiotics (Azithromycin) & Placebo & \\
\hline Currie 1990 & Antibiotics (Amoxicillin) & Placebo & \\
\hline Koh 1997 & Antibiotics (Roxithromycin) & Placebo & $\longleftarrow$ \\
\hline Serisier 2013 & Antibiotics (Ciprofloxacin) & Placebo & \\
\hline Serisier 2013 & Antibiotics (Erythromycin) & Placebo & \\
\hline Wong 2012 & Antibiotics (Azithromycin) & Placebo & $\rightarrow$ \\
\hline Cymbala 2005 & Antibiotics (Azithromycin) & Placebo & - \\
\hline Darke and Know elden 1957 (Oxytetracycline group) & Antibiotics (Oxytetracycline) & Placebo & \\
\hline Darke and Know elden 1957 (Penicilin group) & Antibiotics (Penicillin) & Placebo & \\
\hline Haw orth 2014 & Antibiotics (Colistin) & Placebo & \\
\hline Valery 2013 & Antibiotics (Azithromycin) & Placebo & \\
\hline Barker 2014 (AR-BX 1) & Antibiotics (Aztreonam) & Placebo & \\
\hline Barker 2014 (AR-BX 2) & Antibiotics (Aztreonam) & Placebo & \\
\hline Subtotal ( $($ squared $=58.4 \%, p=0.002)$ & & & $\diamond$ \\
\hline \multicolumn{4}{|l|}{ Inhale d Corticosteroid } \\
\hline Hernando 2012 & Budesonide & Placebo & \\
\hline Tsang 2005 & Fluticasone & Placebo & $\rightarrow$ \\
\hline \multicolumn{4}{|l|}{ Subtotal $($-squared $=0.0 \%, p=0.466$ ) } \\
\hline \multicolumn{4}{|l|}{ Mannitoal } \\
\hline Bilton 2013 & Mannitoal & Placebo & \\
\hline Biton 2014 & Mannitoal & Placebo & $\bullet$ \\
\hline \multicolumn{4}{|l|}{ Subtotal $($ - - squared $=0.0 \%, p=0.863$ ) } \\
\hline \multicolumn{4}{|l|}{ Atorvastatin } \\
\hline Mandal 2014 & Atorvastatin & Placebo & \\
\hline Subtotal (1-squared $=. \%, p=)$. & & & $\infty$ \\
\hline
\end{tabular}

Figure 1 Meta-analysis of number of patients with exacerbations in patients with bronchiectasis who received antibiotics, mucoactive agents, corticosteroids or atorvastatin in comparison to placebo. RR, relative risk. 


\begin{tabular}{|c|c|c|c|c|}
\hline Study ID & Intervention Group & Comparison Group & & \\
\hline \multicolumn{5}{|l|}{ Inhaled corticosteroid } \\
\hline Hernando 2012 & Inhaled corticosteroid & Placebo & $\rightarrow$ & t \\
\hline Tsang 2005 & Inhaled corticosteroid & Placebo & $\rightarrow$ & \\
\hline Subtotal ( 1 squared $=0.0 \%, p=0.941$ ) & & & $d$ & \\
\hline \multicolumn{5}{|l|}{ Mannitoal } \\
\hline Bitton 2013 & Mannitoal & Placebo & & - \\
\hline Bilton 2014 & Mannitoal & Placebo & & + \\
\hline \multicolumn{4}{|l|}{ Subtotal $($-squared $=0.0 \%, p=0.505$ ) } & of \\
\hline \multicolumn{5}{|l|}{ Antibiotics } \\
\hline Cymbala 2005 & Antibiotics (Azithromycin) & Placebo & & \\
\hline de Diego 2013 & Antibiotics (Azithromycin) & Placebo & — & - \\
\hline Orriols 1999 & Antibiotics (Ceftazidime and Tobramycin) & Placebo & - & \\
\hline Masekela 2013 & Antibiotics (Erythromycin) & Placebo & — & - \\
\hline Drobnic 2005 & Antbiotics (Tobramycin) & Placebo & $\rightarrow$ & - \\
\hline Orriols 2015 & Antibiotics (Tobramycin) & Placebo & $\longrightarrow$ & \\
\hline Murray 2011 & Antibiotics (Gentamicin) & Placebo & $\longrightarrow$ & \\
\hline Darke and Know elden 1957 (Penicillin group) & Antibiotics (Penicilitin) & Placebo & - & - \\
\hline Darke and Know elden 1957 (Oxytetracycline group) & Antibiotics (Oxytetracycline) & Placebo & - & - \\
\hline Altenburg 2013 & Antibiotics (Azithromycin) & Placebo & $\longrightarrow$ & \\
\hline Currie 1990 & Antibiotics (Amoxicilin) & Placebo & $\rightarrow-$ & \\
\hline Wong 2012 & Antibiotics (Azithromycin) & Placebo & $\rightarrow$ & \\
\hline Serisier 2013 & Antibiotics (Erythromycin) & Placebo & $\rightarrow$ & \\
\hline Barker 2014 (AIR-BX 1) & Antibiotics (Aztreonam) & Placebo & & + \\
\hline Barker 2014 (AIR-BX 2) & Antibiotics (Aztreonam) & Placebo & & 5 \\
\hline Valery 2013 & Antibiotics (Azithromycin) & Placebo & $\leftarrow$ & \\
\hline Subtotal $($-squared $=81.1 \%, p=0.000)$ & & & $\infty$ & \\
\hline \multicolumn{5}{|l|}{ Mucoactive (rhDNase) } \\
\hline ODonnell 1998 & rhDNase & Placebo & & $\rightarrow$ \\
\hline Subtotal $(k$-squared $=. \%, p=)$. & & & & e \\
\hline NOTE: Weightsare fromrandom effects ana & & & & 0 \\
\hline
\end{tabular}

Figure 2 Meta-analysis of mean number of exacerbations (events) in patients with bronchiectasis who received antibiotics, mucoactive agents or corticosteroids in comparison to placebo. WMD, weighted mean difference.

therapy. The frequency of exacerbations was reduced by longterm antibiotic therapy. Chest PT and PR in breathless patients are routinely recommended by clinicians, but the evidence base to support these recommendations is weak. Bronchiectasis is mostly idiopathic or postinfective. ${ }^{1}$ Identifying an aetiology is recommended by the British Thoracic Society (BTS) Guidelines as there are disease-specific treatments available for bronchiectasis associated with GORD, allergic bronchopulmonary aspergillosis, inflammatory bowel disease, common variable immunodeficiency or environmental mycobacterial infection. ${ }^{1}$ There was insufficient evidence to explore therapies for these conditions in this review.

A key element to patients' management is advising on regular chest clearance. A recent Cochrane review including seven studies with 105 patients concluded that airway clearance techniques appear to be safe for individuals (adults and children) with stable bronchiectasis and may account for improvements in sputum expectoration, selected measures of lung function, symptoms and health-related quality of life. ${ }^{43}$ In our meta-analysis, there were only 29 patients from two studies. ${ }^{39} 40$ Other studies showed improvement in health-related quality of life, with additive benefit with PR in three studies. ${ }^{36-38}$ A systematic review of PR with exercise training in bronchiectasis identified four trials with 164 patients, ${ }^{44}$ concluding that short-term improvements in exercise capacity and health-related quality of life were achieved with supervised PR and exercise training programmes at 8 weeks, but sustaining these benefits long term (ie, $>12$ months) was challenging in these patients. Larger RCTs are needed, as the studies to date are small to make recommendations.
There has been increased interest in mucoactive therapies to aid sputum expectoration. Recent reviews for patients with chronic lung conditions including bronchiectasis concluded that given the harmful effects of DNase and insignificant evidence of benefit, DNase should not be used in routine practice. ${ }^{45} 46$ There is insufficient evidence to recommend the routine use of other mucolytics in bronchiectasis. ${ }^{45} 46$ In our meta-analysis, there was no benefit with inhaled recombinant human DNase, which supports BTS recommendations against its use in bronchiectasis. ${ }^{1}$ The main study of inhaled mannitol has shown increased time to next exacerbation and reduced antibiotic exposure but failed to reach its primary endpoint of reduced exacerbations. The downsides include the treatment burden of inhaling 10 capsules twice daily and the fact that about $22 \%$ of patients are unable to tolerate mannitol with the development of significant bronchospasm with a greater than $20 \%$ fall in $\mathrm{FEV}_{1}$. Overall mannitol is unlikely to be licensed for routine use in bronchiectasis.

There are novel anti-inflammatory therapies that can potentially reduce exacerbations. Atorvastatin reduced exacerbations in patients with moderate to severe bronchiectasis in a singlecentre study. The study showed improved neutrophil apoptosis, postulating a potential mechanism about how statins led to clinical improvement; 33\% had however non-serious AEs versus $10 \%$ taking placebo. Multicentred studies are needed to address whether such therapy is tolerable and can reduce exacerbations before it can be recommended for routine use.

A 2009 review showed insufficient evidence to recommend the routine use of inhaled corticosteroids in adults with stable 
bronchiectasis. ${ }^{47}$ Our analysis only included 163 eligible patients for the efficacy of inhaled corticosteroids, showing no effect on exacerbations, hospitalisation or mortality. There is insufficient evidence to support the routine use of inhaled corticosteroids in bronchiectasis. The exceptions are in patients with coexisting asthma or severe chronic obstructive pulmonary disease (COPD) with frequent exacerbations that merit inhaled corticosteroids for these conditions alone from international guidelines.

Regarding long-term antibiotics, a Cochrane review in 2015 evaluating treatments given during a period of 4-83 weeks found at least a 50\% reduction in exacerbations and hospitalisation. The risk of developing drug resistance was more than threefold higher. The review was however limited by the diversity and evidence of moderate to low quality. ${ }^{48}$ A more targeted meta-analysis investigating inhaled antibiotic therapy taken for 4 weeks or longer in bronchiectasis found that inhaled antibiotics reduced the bacterial load, enhanced bacterial clearance and reduced the risk of acute exacerbations. ${ }^{49}$ In a meta-analysis of long-term macrolides, the authors included 10 studies containing 601 patients where macrolides led to a statistically significant improvement in reducing acute exacerbations per patient during follow-up treatment $(\mathrm{p}<0.001)$, increasing the number of patients free from exacerbations $(p<0.001)$ and prolonging time to a first exacerbation $(p<0.001)$. Macrolide maintenance treatment was superior to control with respect to attenuating $\mathrm{FEV}_{1}$ decline $(\mathrm{p}=0.02)$, improving sputum volume $(\mathrm{p}=0.009)$ and SGRQ total scores $(\mathrm{p}=0.02)$, but showed a higher risk of AEs, especially diarrhoea $(p=0.0006)$. Eradication of pathogens did not significantly improve in the macrolide group ( $p=0.09$ ), while pathogen resistance caused by macrolides increased $(\mathrm{p}<0.001){ }^{50}$

In our meta-analysis investigating treatments taken for 3 months or longer, we were able to summarise evidence from only 1196 patients on the efficacy of long-term antibiotic therapy. It gave a clear signal that long-term antibiotics reduce sputum purulence and exacerbations. There was no signal whether there was any effect on hospitalisation and mortality. Larger and longer RCTs are needed to explore these endpoints along with tolerability. We analysed whether the positive effect on reducing exacerbations was dependent on route of administration (oral or inhaled) and if on long-term oral antibiotics whether there was different efficacy if on long-term macrolide therapy or not, but this review showed no significant differences; however, there have been no head to head trials comparing inhaled antibiotics versus oral antibiotics. Similar to Fan et $a l,{ }^{50}$ there was increased diarrhoea with long-term macrolide therapy. It is well recognised that inhaled therapies can lead to chest pain, wheeze and breathlessness due to local bronchospasm. The meta-analysis by Brodt and colleagues reported that 10\% have bronchospasm with inhaled antibiotics compared with $2.3 \%$ placebo. ${ }^{49}$ Similarly, in our study, there was increased risk of bronchospasm with inhaled antibiotics and a small reduction in $\mathrm{FVC} \%$. Although there were no significant differences identified between oral and inhaled antibiotics in this review, we would interpret these results with caution in light of the small sample size. Further RCTs are needed but there is a clear signal for the benefit of long-term antibiotics. Further work is needed to determine the optimal bronchiectasis patients we should consider for long-term antibiotic therapy, the agent to be used, route of administration and length of treatment, and we need long-term follow-up to assess the long-term safety of these agents, in particular the concerns of developing drug resistance and the development of emergent new pathogens.

The limitations of this evidence synthesis include heterogeneity between studies in defining exacerbations (various protocols), variations in the modalities used to treat patients, insufficient or unclear reporting of clinical outcomes of interest and limited inference of patients' disease severity. The strengths of this review include expansive inclusion of clinically relevant pharmacological and non-pharmacological interventions, robust methodology in synthesising and determining the strength of evidence per intervention, the input of panel expert, the analysis of well-defined treatments and ascertaining the time period for each intervention to minimise biases.

At the time of this review, only 30 studies, mostly studies on adult patients, were available to guide the care of patients with this condition. This is in comparison to a PubMed search, dated 23 November 2017, in which there were 5080 randomised controlled studies on COPD, 7533 on lung cancer and 10901 on asthma. This review confirms the paucity of RCTs in bronchiectasis. Larger RCTs are needed to assess the role of chest clearance and PR in improving exacerbations as the studies to date are underpowered to make recommendations. The evidence warranting most confidence is for long-term antibiotic therapy; however, there is insufficient evidence to identify the optimum agent, mode of therapy and length of treatment. Large multicentre double-blind trials are needed to address these short-term and long-term treatment strategies in bronchiectasis.

Contributors All authors contributed significantly to this work. Work conception by ATH and MHM. Data search, crossreferencing, screening and extraction by AMAD, NA, QH, WHF, $\mathrm{KB}$, LJP and CCD. Data analysis by AMAD, ATH, and ZW. Manuscript writing by AMAD, ATH, MHM and CCD. Manuscript revision and approval by all coauthors. AMAD, ATH and MHM take responsibility for the integrity of the work as a whole, from inception to published article.

Competing interests ATH has been on advisory boards for Bayer, Griffolls and Pfizer.

Patient consent Detail has been removed from this case description/these case descriptions to ensure anonymity. The editors and reviewers have seen the detailed information available and are satisfied that the information backs up the case the authors are making.

Provenance and peer review Not commissioned; externally peer reviewed.

( Article author(s) (or their employer(s) unless otherwise stated in the text of the article) 2018. All rights reserved. No commercial use is permitted unless otherwise expressly granted.

\section{References}

1. Pasteur MC, Bilton D, Hill AT, et al. British Thoracic Society guideline for non-CF bronchiectasis. Thorax 2010;65:577-i58.

2. Weycker D, Edelsberg J, Oster G, et al. Prevalence and Economic Burden of Bronchiectasis. Clin Pulm Med 2005;12:205-9.

3. Martínez-García MÁ, de Gracia J, Vendrell Relat M, et al. Multidimensional approach to non-cystic fibrosis bronchiectasis: the FACED score. Eur Respir J 2014;43:1357-67.

4. Chalmers JD, Goeminne P, Aliberti S, et al. The bronchiectasis severity index. An international derivation and validation study. Am J Respir Crit Care Med 2014;189:576-85.

5. Angrill J, Agusti C, De Celis R, et al. Bronchial inflammation and colonization in patients with clinically stable bronchiectasis. Am J Respir Crit Care Med 2001;164:1628-32.

6. Chalmers JD, Smith MP, McHugh BJ, et al. Short- and long-term antibiotic treatment reduces airway and systemic inflammation in non-cystic fibrosis bronchiectasis. Am J Respir Crit Care Med 2012;186:657-65.

7. Liberati A, Altman DG, Tetzlaff J, et al. The PRISMA statement for reporting systematic reviews and meta-analyses of studies that evaluate 
health care interventions: explanation and elaboration. PLoS Med 2009;6:e1000100

8. Jones PW, Quirk FH, Baveystock CM, et al. A self-complete measure of health status for chronic airflow limitation. The St. George's Respiratory Questionnaire. Am Rev Respir Dis 1992;145:1321-7.

9. Barr JT, Schumacher GE, Freeman S, et al. American translation, modification, and validation of the St. George's Respiratory Questionnaire Clin Ther 2000;22:1121-45.

10. Murray MP, Turnbull K, MacQuarrie S, et al. Validation of the Leicester Cough Questionnaire in non-cystic fibrosis bronchiectasis. Eur Respir J 2009;34:125-31.

11. Higgins JP, Altman DG, Gøtzsche PC, et al. The Cochrane Collaboration's tool for assessing risk of bias in randomised trials. BMJ 2011;343:d5928.

12. Guyatt GH, Oxman AD, Schünemann HJ, et al. GRADE guidelines: a new series of articles in the Journal of Clinical Epidemiology. J Clin Epidemiol 2011;64:380-2.

13. Masekela R, Anderson R, Gongxeka H, et al. Lack of efficacy of an immunomodulatory macrolide in childhood HIV related bronchiectasis: A randomised, placebo-controlled trial. Journal of Antivirals and Antiretrovirals 2013;5:044-9.

14. Koh YY, Lee MH, Sun YH, et al. Effect of roxithromycin on airway responsiveness in children with bronchiectasis: a double-blind, placebocontrolled study. Eur Respir J 1997;10:994-9.

15. Valery PC, Morris PS, Byrnes CA, et al. Long-term azithromycin for Indigenous children with non-cystic-fibrosis bronchiectasis or chronic suppurative lung disease (Bronchiectasis Intervention Study): a multicentre, double-blind, randomised controlled trial. Lancet Respir Med 2013;1:610-20

16. Altenburg J, de Graaff CS, Stienstra Y, et al. Effect of azithromycin maintenance treatment on infectious exacerbations among patients with non-cystic fibrosis bronchiectasis: the BAT randomized controlled trial. JAMA 2013;309:1251-9.

17. Barker AF, O'Donnell AE, Flume $\mathrm{P}$, et al. Aztreonam for inhalation solution in patients with non-cystic fibrosis bronchiectasis (AIR-BX1 and AIR-BX2): two randomised double-blind, placebo-controlled phase 3 trials. Lancet Respir Med 2014;2:738-49.

18. Currie DC, Garbett ND, Chan KL, et al. Double-blind randomized study of prolonged higher-dose oral amoxycillin in purulent bronchiectasis. Q $J$ Med 1990;76:799-816.

19. Cymbala AA, Edmonds LC, Bauer MA, et al. The disease-modifying effects of twice-weekly oral azithromycin in patients with bronchiectasis. Treat Respir Med 2005;4:117-22.

20. Diego AD, Milara J, Martinez-Moragón E, et al. Effects of long-term azithromycin therapy on airway oxidative stress markers in non-cystic fibrosis bronchiectasis. Respirology 2013;18:1056-62.

21. Drobnic ME, Suñé P, Montoro JB, et al. Inhaled tobramycin in noncystic fibrosis patients with bronchiectasis and chronic bronchial infection with Pseudomonas aeruginosa. Ann Pharmacother 2005;39:39-44.

22. Haworth CS, Foweraker JE, Wilkinson P, et al. Inhaled colistin in patients with bronchiectasis and chronic Pseudomonas aeruginosa infection. Am J Respir Crit Care Med 2014;189:975-82.

23. Murray MP, Govan JR, Doherty CJ, et al. A randomized controlled trial of nebulized gentamicin in non-cystic fibrosis bronchiectasis. Am J Respir Crit Care Med 2011;183:491-9.

24. Orriols R, Roig J, Ferrer J, et al. Inhaled antibiotic therapy in non-cystic fibrosis patients with bronchiectasis and chronic bronchial infection by Pseudomonas aeruginosa. Respir Med 1999;93:476-80.

25. Serisier DJ, Bilton D, De Soyza A, et al. Inhaled, dual release liposomal ciprofloxacin in non-cystic fibrosis bronchiectasis (ORBIT-2): a randomised, double-blind, placebo-controlled trial. Thorax 2013;68:812-7.

26. Serisier DJ, Martin ML, McGuckin MA, et al. Effect of long-term, low-dose erythromycin on pulmonary exacerbations among patients with non-cystic fibrosis bronchiectasis: the BLESS randomized controlled trial. JAMA 2013;309:1260-7.

27. Wong C, Jayaram L, Karalus N, et al. Azithromycin for prevention of exacerbations in non-cystic fibrosis bronchiectasis (EMBRACE): a randomised, double-blind, placebo-controlled trial. Lancet 2012;380:660-7.

28. CaKJ D. PROLONGED antibiotic treatment of severe bronchiectasis; a report by a subcommittee of the Antibiotics Clinical Trials (nontuberculous) Committee of the Medical Research Council. Br Med J 1957;2:255-9.

29. Orriols R, Hernando R, Ferrer A, et al. Eradication Therapy against Pseudomonas aeruginosa in Non-Cystic Fibrosis Bronchiectasis. Respiration 2015;90:299-305.

30. Bilton D, Daviskas E, Anderson SD, et al. Phase 3 randomized study of the efficacy and safety of inhaled dry powder mannitol for the symptomatic treatment of non-cystic fibrosis bronchiectasis. Chest 2013;144:215-25.

31. Bilton D, Tino G, Barker AF, et al. Inhaled mannitol for non-cystic fibrosis bronchiectasis: a randomised, controlled trial. Thorax 2014;69:1073-9.

32. O'Donnell AE, Barker AF, Ilowite JS, et al. Treatment of Idiopathic Bronchiectasis With Aerosolized Recombinant Human DNase I. Chest 1998;113:1329-34.

33. Hernando R, Drobnic ME, Cruz MJ, et al. Budesonide efficacy and safety in patients with bronchiectasis not due to cystic fibrosis. Int J Clin Pharm 2012;34:644-50.

34. Tsang KW, et al. Inhaled fluticasone in bronchiectasis: a 12 month study. Thorax 2005;60:239-43.

35. Martínez-García MA, Perpiñá-Tordera M, Román-Sánchez P, et al. Inhaled steroids improve quality of life in patients with steady-state bronchiectasis. Respir Med 2006;100:1623-32.

36. Mandal P, Sidhu MK, Kope L, et al. A pilot study of pulmonary rehabilitation and chest physiotherapy versus chest physiotherapy alone in bronchiectasis. Respir Med 2012;106:1647-54.

37. Lee AL, Hill CJ, Cecins N, et al. The short and long term effects of exercise training in non-cystic fibrosis bronchiectasis - a randomised controlled trial. Respir Res 2014;15:44.

38. Newall C, Stockley RA, Hill SL. Exercise training and inspiratory muscle training in patients with bronchiectasis. Thorax 2005;60:943-8.

39. Figueiredo PHS, Zin WA, Guimarães FS. Flutter valve improves respiratory mechanics and sputum production in patients with bronchiectasis. Physiotherapy Research International 2012;17:12-20.

40. Murray MP, Pentland JL, Hill AT. A randomised crossover trial of chest physiotherapy in non-cystic fibrosis bronchiectasis. Eur Respir J 2009;34:1086-92.

41. Mandal P, Chalmers JD, Graham C, et al. Atorvastatin as a stable treatment in bronchiectasis: a randomised controlled trial. Lancet Respir Med 2014;2:455-63.

42. Martínez-García Miguel Ángel, Soler-Cataluña JJ, Catalán-Serra P, et al. Clinical Efficacy and Safety of Budesonide-Formoterol in Non-Cystic Fibrosis Bronchiectasis. Chest 2012;141:461-8.

43. Lee AL, Burge AT, Holland AE. Airway clearance techniques for bronchiectasis. Cochrane Database Syst Rev 2015;11:CD008351.

44. Lee AL, Hill CJ, McDonald CF, et al. Pulmonary Rehabilitation in Individuals With Non-Cystic Fibrosis Bronchiectasis: A Systematic Review. Arch Phys Med Rehabil 2016.

45. Wilkinson M, Sugumar K, Milan SJ, et al. Mucolytics for bronchiectasis. Cochrane Database Syst Rev 2014;29:CD001289.

46. Tarrant BJ, Le Maitre C, Romero L, et al. Mucoactive agents for chronic, non-cystic fibrosis lung disease: A systematic review and meta-analysis. Respirology 2017;22:1084-92.

47. Kapur N, Bell S, Kolbe J, et al. Inhaled steroids for bronchiectasis. Cochrane Database Syst Rev 2009;86:CD000996.

48. Hnin K, Nguyen C, Carson KV, et al. Prolonged antibiotics for non-cystic fibrosis bronchiectasis in children and adults. Cochrane Database Syst Rev 2015;209:CD001392.

49. Brodt AM, Stovold E, Zhang L. Inhaled antibiotics for stable non-cystic fibrosis bronchiectasis: a systematic review. Eur Respir J 2014;44:382-93.

50. Fan L-C, Lu H-W, Wei P, et al. Effects of long-term use of macrolides in patients with non-cystic fibrosis bronchiectasis: a meta-analysis of randomized controlled trials. BMC Infect Dis 2015;15:160. 